experiments support this view: for example, if the engrailed gene is removed from the $\mathrm{P}$ cells, the cells make structures characteristic of the A compartment (that is, they acquire A identity); they also gain different affinities and they sort out from their neighbours, even forming vesicles in some cases. If such cells are made near to the $\mathrm{A} / \mathrm{P}$ border they can sort into the A compartment and define the border from the anterior side. So there is little doubt that engrailed controls the differences between $\mathrm{A}$ and $\mathrm{P}$ cells, including their affinities.

The two groups ${ }^{2,3}$ now delve into the question of whether engrailed specifies affinities directly or indirectly. In the growing imaginal wing disk, $\mathrm{P}$ cells signal by means of Hedgehog protein (Hh), which instructs the nearby A cells to turn on synthesis of Decapentaplegic (Dpp), a longrange morphogen; $\mathrm{Hh}$ also gives the cells positional information by telling them that they are near the border (see ref. 7). The experimental method in both papers is the same, and makes use of the smoothened gene; this gene encodes a component of the Hh receptor which the cells depend upon to see $\mathrm{Hh}$ (ref. 8). Flies are made in which the sister cells from a single division can be individually marked to generate a pair of marked clones, a 'twin spot'. One sister clone lacks the smoothened gene $\left(s m o^{-}\right)$, and the other $\left(s m o^{+}\right)$is wild type (Fig. 1a).

Both papers include beautiful pictures showing that when a twin spot is made in the A compartment, near the boundary, the smoclone, which retains A identity, nevertheless moves backwards into $\mathrm{P}$ territory, while its sister $\mathrm{smo}^{+}$clone stays in the A domain (Fig. 1b). Moreover the $s m o^{-}$clone now forms a straight edge against the other A cells, close to the normal position of the A/P boundary. This is a remarkable finding; it suggests that the largest contributor to the affinity difference between $\mathrm{A}$ and $\mathrm{P}$ cells, at least in the region of the $\mathrm{A} / \mathrm{P}$ boundary, is not Engrailed acting directly on the $\mathrm{P}$ cells, but is an indirect result of $\mathrm{Hh}$ signalling from $\mathrm{P}$ to $\mathrm{A}$ cells across the border.

There is, however, a point of difference between the two sets of results. Rodriguez and Basler show the smo- clones forming a reasonably wiggly boundary with their new $\mathrm{P}$ neighbours in the imaginal disk, whereas Blair and Ralston look at the pupal wing and find that the displaced $s m o^{-}$clone has rather straight boundaries with both A and P neighbours. These results then take the authors in different directions: Rodriguez and Basler argue that Engrailed acts indirectly on affinities entirely, or almost entirely, through the Hh signal; Blair and Ralston suggest that, in addition, Engrailed may act autonomously to give a distinct affinity to $P$ cells.

To this mix I would add one point. There is some evidence that affinities are graded along the antero-posterior axis ${ }^{9}$, making the positional identity of the $s m o^{-}$cells crucial to the argument. One would expect that the mutant cells, not receiving any $\mathrm{Hh}$, would 'believe' they were at the anterior edge of the A compartment - that is, remote from the Hh source in situ and, therefore, also remote from the peak of the Dpp gradient. In the adult abdomen of Drosophila, smo clones located in an equivalent position do indeed make the type of cuticle found remote from the A/P boundary (ref. 10, Figs 6B, 7).

If the $\mathrm{Hh}$ signal were the only effector of affinity, the smo cells, receiving no signal, should retain the same affinity as the P cells, and form a wiggly boundary with them, and this is what Rodriguez and Basler show in the disk. By contrast, Blair and Ralston's results show that the $s m o^{-}$cells form straight boundaries with both $\mathrm{A}$ and $\mathrm{P}$ neighbours in the pupal wing, and this also happens in the abdomen. These observations seem to support each other, and they suggest that the $\mathrm{Hh}$ signal is not the whole story and that there is probably a difference in quality between $\mathrm{A}$ and $\mathrm{P}$ affinities.

Chemotaxis

\section{On the crest of a spiral wave}

Drop a pebble into a pool of water and you'll see a series of concentric waves, fanning outwards in ever-increasing circles. Subject slime moulds on a surface to starvation and a similar effect occurs — single-celled Dictyostelium discoideum amoebae move chemotactically towards the periodic waves of cyclic AMP that are emitted from the so-called aggregation centre.

Reporting in Proceedings of the National Academy of Sciences, Jacques Lauzeral and his colleagues have looked at these cAMP waves and, in particular, at the spiral waves that develop from broken circular waves (Proc. Natl Acad. Sci. USA 94, 9153-9158; 1997). By studying the developmental path along which the slime moulds travel, the authors believe that that they can now explain how these spiral waves form.

The aggregation centre acts as a 'pacemaker', generating a pulse of cAMP at five- to ten-minute intervals. Under conditions of starvation the amoebae become excitable and, on binding cAMP, an enzyme called adenylate cyclase is activated. This leads to the production of more CAMP, thereby propagating the wave. But, eventually, the amoebae become desensitized to cAMP — they stop binding it, and the wave recedes while the amoebae recover their ability to sense and relay the approaching wave.

Where do the spiral waves come from? Lauzeral et al. claim that the key is desynchronization of cells on the developmental
The interpretation I prefer is therefore that there are at least two contributions to cell affinity — a main one (perhaps graded) related to the positional information that is initiated by and consequent on the $\mathrm{Hh}$ signal, and also a difference between A and $\mathrm{P}$ that is dependent more directly on Engrailed.

Peter A. Lawrence is in the MRC Laboratory of Molecular Biology, Hills Road, Cambridge

CB2 2QH, UK.

e-mail:pal@mrc-lmb.cam.ac.uk
Garcia-Bellido, A. in Biology of Imaginal Discs (eds Ursprung
H. \& Nothiger, R.) 59-91 (Springer, Heidelberg, 1972).
2. Blair, S. S. \& Ralston, A. Development 124, 4053-4063
(1997)
3. Rodriguez, I. \& Basler, K. Nature 389, 614-618 (1997).
4. Townes, P. L. \& Holtfreter, J. J. Exp. Zool. 128, 53-120 (1955).
5. Morata, G. \& Garcia-Bellido, A. Wilhelm Roux Arch. 179, 125-143 (1976).
6. Morata, G. \& Lawrence, P. A. Nature 255, 614-617 (1975).
Mullor, J. L., Calleja, M., Capdevila, J. \& Guerrero, I. Development 124, 1227-1237 (1997).
8. Chen, Y. \& Struhl, G. Cell 87, 553-563 (1996)
9. Wright, D. A. \& Lawrence, P. A. Dev. Biol. 85, 317-327 (1981).
10. Struhl, G., Barbash, D. \& Lawrence, P. A. Development 124, 2155-2165 (1997) 